\title{
marges Marges
}

revue d'art contemporain Revue d'art contemporain

$01 \mid 2003$

Varia

\section{«Les ombres et les survivances chez Mimmo}

\section{Paladino à travers Homère »}

"The Shadow and Survivances in Mimmo Paladino's Iliad and Ulysses"

\section{Soko Phay-Vakalis}

\section{(2) OpenEdition}

\section{Journals}

Édition électronique

URL : http://journals.openedition.org/marges/827

DOI : $10.4000 /$ marges. 827

ISSN : 2416-8742

\section{Éditeur}

Presses universitaires de Vincennes

\section{Édition imprimée}

Date de publication : 15 mars 2003

Pagination : 78-93

ISBN : 978-2-84292-245-0

ISSN : $1767-7114$

\section{Référence électronique}

Soko Phay-Vakalis, « « Les ombres et les survivances chez Mimmo Paladino à travers Homère » », Marges [En ligne], 01 | 2003, mis en ligne le 15 mars 2004, consulté le 20 avril 2019. URL : http:// journals.openedition.org/marges/827 ; DOI : 10.4000/marges.827 


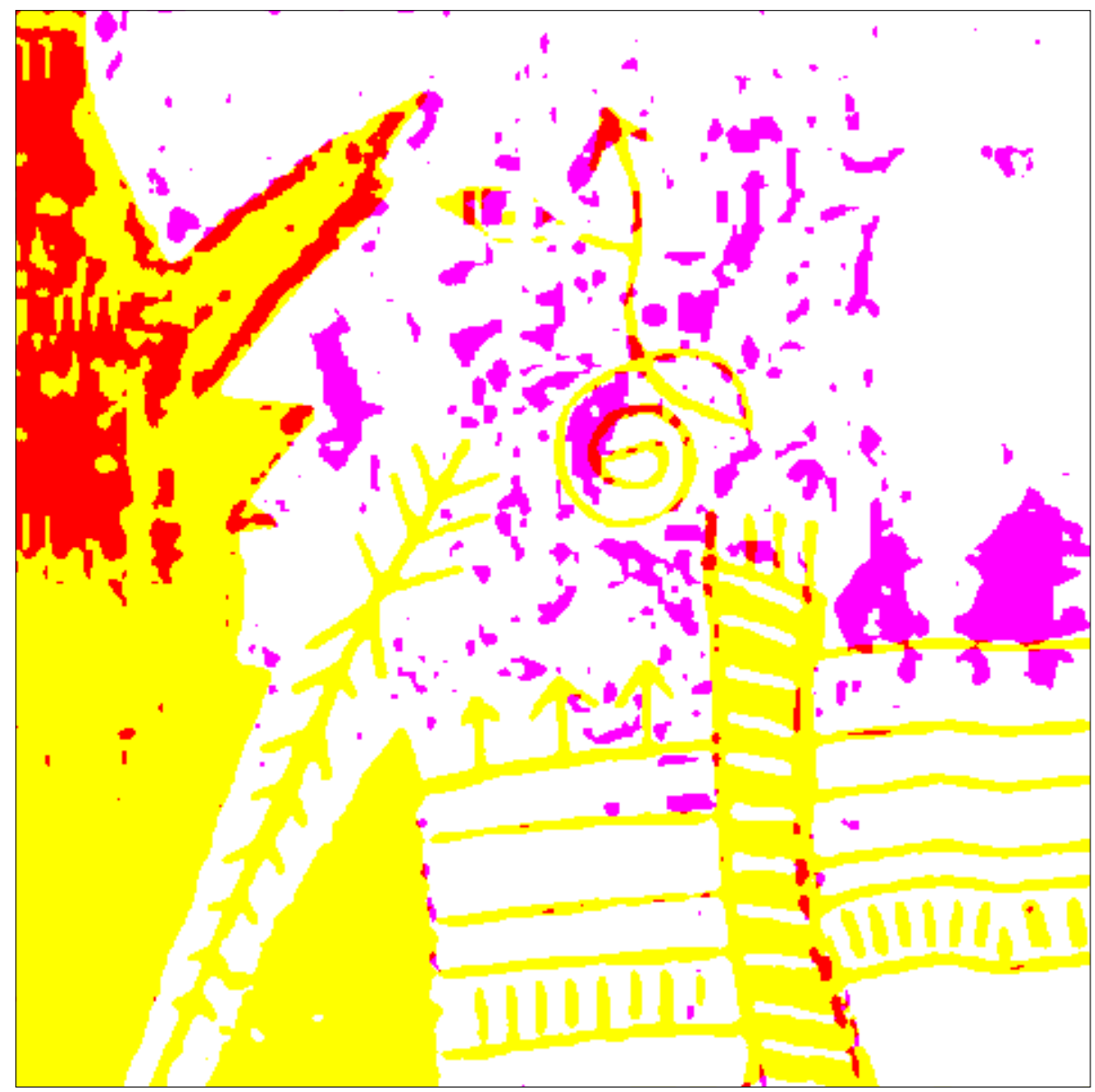




\section{« Les ombres et les survivances chez Mimmo Paladino à travers Homère ${ }^{*}$}

La sortie de l'lliade et de l'Odyssée, illustrées par Mimmo Paladino en septembre 2001 et accompagnées d'une exposition sur le même thème en automne de la même année à la Galerie Thaddaeus Ropac de Paris a été annoncée comme un événement culturel important. En effet, pour la première fois en 2700 ans un peintre illustre les épopées d'Homère dans leur totalité ; c'est Mimmo Paladino, né en 1948 en Italie du Sud qui a été choisi par l'éditrice Diane de Selliers, notamment pour sa culture méditerranéenne où se mêlent les influences étrusque, égyptienne, grecque, romaine et chrétienne. Deux cent deux œuvres accompagnent les textes. L'artiste exclut toute illustration servile : " Je pars de la peinture pour arriver à une histoire pour accéder à la peinture. Mes images ne veulent pas raconter des histoires, même si parfois elles donnent cette impression. Il n'y a pas d'explication adéquate possible, seulement des allusions. Les éléments de l'image ne racontent rien. Ils se rapprochent ou s'opposent, ils reviennent ; la conception est plutôt musicale, de toute façon elle n'est pas littéraire ${ }^{1}$ " confie-t-il. Pendant deux années, Paladino s'est exprimé à travers une palette très large de techniques : crayon, gouache, encre de Chine, grattage, brûlage, feuille d'or...
* Communication prononcée en novembre 2002, dans le cadre du colloque « Homère virtuel pour un "compagnon" électronique aux études homériques », organisé par le Centre de recherches homériques de l'Université Stendhal, Grenoble 3, sous la responsabilité de Françoise Létoublon.

${ }^{1}$ Mimmo Paladino, cité dans le communiqué de presse de la Galerie Thaddaeus Ropac/Diane de Selliers, Paris, 2001.

Page 78

Mimmo Paladino, détail, couverture du coffret Homère pour les éditions Diane de Selliers, Paris, 2001. 
2 Bernard LamarcheVadel, Mimmo Paladino, le guetteur, Galerie Beaubourg/ La Différence, Paris, 1988, p. 8.

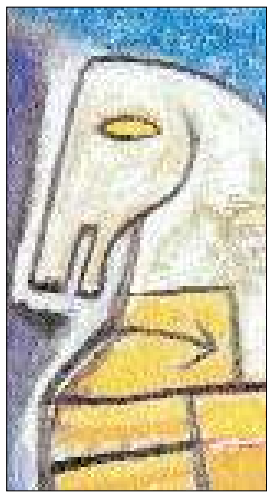

Détail, $4^{\mathrm{e}}$ de couverture, p. 87.

\section{Page 81}

Mimmo Paladino, $4^{\mathrm{e}}$ de couverture du coffret Homère pour les éditions Diane de Selliers, Paris, 2001.

Analyser le travail de Paladino sur Homère permettra de comprendre comment une création contemporaine peut rendre compte d'une œuvre littéraire vieille de plusieurs millénaires. De quelles manières peut-on parler d'« images survivantes ", pour emprunter le concept de Nachleben d'Aby Warburg, chez Mimmo Paladino ?

\section{L'anamnèse comme esthétique de}

\section{la trans-avant-garde}

La trans-avant-garde à laquelle sont liés Mimmo Paladino, Francesco Clemente, Sandro Chia et Enzo Cucchi, est apparue dès le début des années quatre-vingt. Elle s'est voulue - et a été perçue - comme une véritable renaissance de la tradition. Au-delà des différentes inventions formelles, s'est instaurée une conception inédite de la peinture comme un lieu de "morceaux choisis" de cultures hétéroclites. Il s'agit de saisir dans les œuvres de Paladino les traces d'une culture, en l'occurrence méditerranéenne. Comment traitent-elles de la mémoire : en remodelant le passé ou en élaborant sa filiation sous formes d'apparents retours en arrière ? Comment Paladino se réapproprie-t-il les mouvements artistiques antérieurs tout en les offrant au regard du spectateur sous un jour nouveau ? C'est là une manière de se défier de toute notion d'avant-garde ou de progrès en art, et l'œuvre de Paladino n'a de cesse de nous le prouver depuis les années quatre-vingt, et particulièrement à travers ses illustrations d'Homère.

l'emprunte le concept de "l'anamnèse » qui est au centre de l'expérience artistique et esthétique de la trans-avant-garde pour définir ma méthodologie. Dans sa présentation de l'œuvre de Paladino, Bernard Lamarche-Vadel en donne la définition : "L'anamnèse est à la fois une technique, une ligne temporelle et un espace. Une technique, puisque l'anamnèse en terme médical, consiste en une enquête sur toutes les circonstances d'états antérieurs susceptibles d'éclairer l'état actuel d'un patient. De ce fait l'anamnèse est une dynamique de la successivité réversible. Plus ou moins éloignés dans le passé, mais reliés entre eux, les souvenirs forment une chaîne temporelle marquée aussi bien de régressions que de progressions en regard du moment où l'énoncé dispose les étapes du processus menant au présent. Espace enfin, l'anamnèse, en sa qualité de dispositif de signes inscrits dans toutes les positions imaginables à l'intérieur d'une structure figurale profonde ${ }^{2}$. " En outre, à l'instar de l'Odyssée qui ne suit pas d'ordre chronologique, mais les errances de la mémoire (notamment par une série 


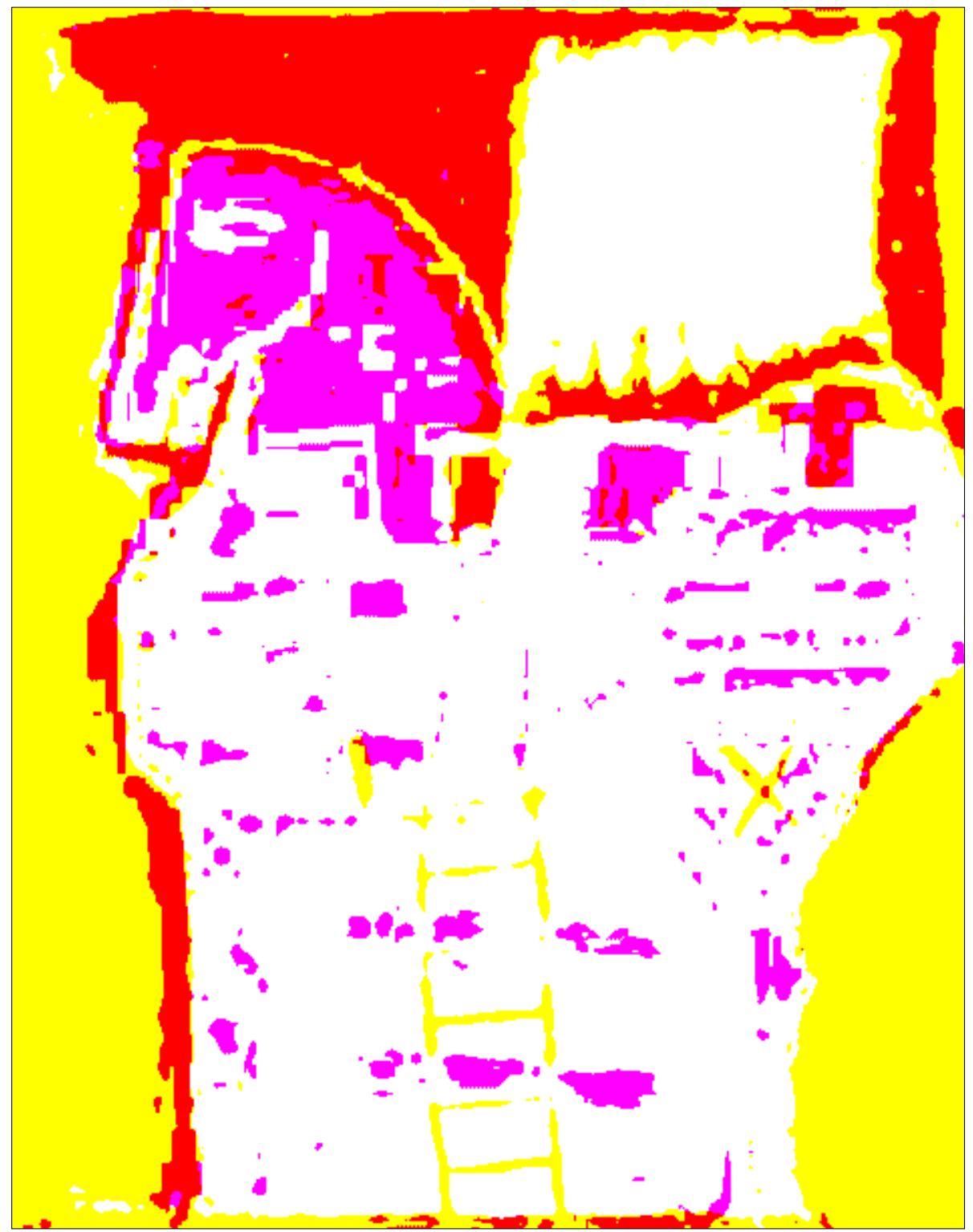


de retours en arrière où les sept ans dans l'île de Calypso tiennent moins de place qu'une nuit chez le Cyclope), je confronterai à la fois des motifs anciens et contemporains, des idées ou concepts de l'époque d'Homère et d'aujourd'hui. Cette lecture transversale des cultures artistiques et des époques permettra de dégager l'originalité de l'art de Paladino et de comprendre l'enjeu de la peinture contemporaine : le langage artistique est alors admis comme un instrument de transition, de passage d'une œuvre à l'autre, d'un style à l'autre. En adoptant une attitude nomade de réversibilité des langages du passé, Paladino s'oppose aux différents mythes modernistes qui croient en la rupture avec passé et en le progrès de l'art. Enfin, il inverse l'idée d'une linéarité de l'histoire de l'art.

\section{L'image survivante comme fragment}

En réaction contre la lassitude à l'égard du purisme et de l'austérité de l'Art Minimal, du manque de sensualité de l'Art Conceptuel, les artistes de la Trans-avant-garde revendiquent un fort enracinement dans la tradition et reviennent à un art construit avec des moyens d'expression multiples, et plus particulièrement avec la matière même de la peinture, c'est-à-dire avec les instruments liés au langage des signes et de la couleur.

Dans le rejet d'une peinture autonome conçue comme une fin en soi, celle de Paladino se réfère au présent et interroge également l'histoire et les mythes. De par leur charge symbolique subjective, ses tableaux admettent différentes interprétations. En travaillant sur le fragment - symptôme de la dissociation et d'un désir d'une continuelle mutation -, Paladino revient à la conception de la position centrale de l'art, qui devient le lieu de confluence et d'émergence de la sensibilité. La peinture cristallise, à l'intérieur d'ellemême, la mémoire culturelle et visuelle d'autres œuvres, non pas à titre de citations mais en qualité de construction mobile, c'est-àdire dynamique, énergétique. Ainsi, le fragment révèle qu'une image peut se construire, à travers la subjectivité de l'artiste, par bonds hors d'un projet linéaire. Émergence et persistance seraient les caractéristiques des images de Paladino qui témoignent à la fois de l'héritage des procédés traditionnels de l'art, des cultures anciennes ou mineures et de sa capacité à réaliser des écarts et des différences propres à la notion d'avant-garde.

À travers quelques analyses iconographiques, je montrerai comment Paladino procède par « évocation » comme affirmation d'une volonté d'anamnèse. Toutefois, ces évocations sont rarement isolées. Elles adviennent dans une trame très serrée de styles repris et 
métamorphosés dont le spectateur retrouve difficilement l'origine précise. Leur entrecroisement provoque un sentiment intense et déroutant de déjà vu. Ainsi les éléments repérés sont à la fois omniprésents et « brouillés".

Dans les illustrations d'Homère, on peut repérer certaines récurrences : les tables couvertes de mets, l'Olympe et les apparitions divines, les animaux (ceux des présages, des métamorphoses ou des attelages), etc. La représentation humaine est aussi très importante. Les personnages sont droits et immobiles. Leur expressivité dépend de l'attitude. Les gestes donnent le mouvement et définissent l'action. Depuis que Paladino exécute des peintures figuratives - dès le début des années quatre-vingt - il accorde une place prépondérante au corps humain. Il s'engage peu à peu dans une voie qui l'a progressivement conduit à concentrer son attention sur des figures hiératiques ou totémiques. Leur archaïsme se teinte à la fois de classicisme et de mysticisme à l'instar des sculptures blanches.

Devant la variété des motifs de Paladino dans l'lliade et l'Odyssée, j'ai choisi de travailler, sur la question de l'ombre dans la représentation humaine. En quoi l'ombre est-elle un motif de réflexion tant à l'époque d'Homère qu'à celle de Paladino ? De quelles manières détermine-t-elle l'esthétique de Paladino ?

\section{Les silhouettes fantomatiques}

Chez Paladino, les représentations humaines ressemblent davantage à des ombres, des silhouettes fantomatiques, notamment par son utilisation de l'aquarelle ou de l'encre de Chine. Parmi les personnages, certains sont mutilés, cernés par un trait de couleur ou encore vidés de leur chair, comme l'illustration de l'lliade page 48. Assemblés sur plusieurs registres ; ils font office de "veilleurs ». D'autres exemples des pages 325, 334 et 339 de l'lliade montrent que les figures sont représentées au même degré de réalité. En effet, le spectateur ne peut faire la différence entre les dieux et les mortels - en particulier entre les morts et les vivants - seulement par l'action ou l'immobilité des corps. Chez Paladino, les registres des mondes des cieux ou des enfers fusionnent en un monde de spectres, d'âmes errantes.

Dans la Grèce archaïque, la figure humaine sur les vases géométriques apparaît au VIIIe siècle avant notre ère. Sa représentation n'est apparemment précédée d'aucun essai isolé. Au contraire, les personnages en silhouette noire sont nombreux, démultipliés, mis en scène, occupant des surfaces considérables ${ }^{3}$. 
Mimmo Paladino, illustration de l'lliade, Chant II, 2001.

Mimmo Paladino, illustration de l'/liade, Chant XXIV, 2001.
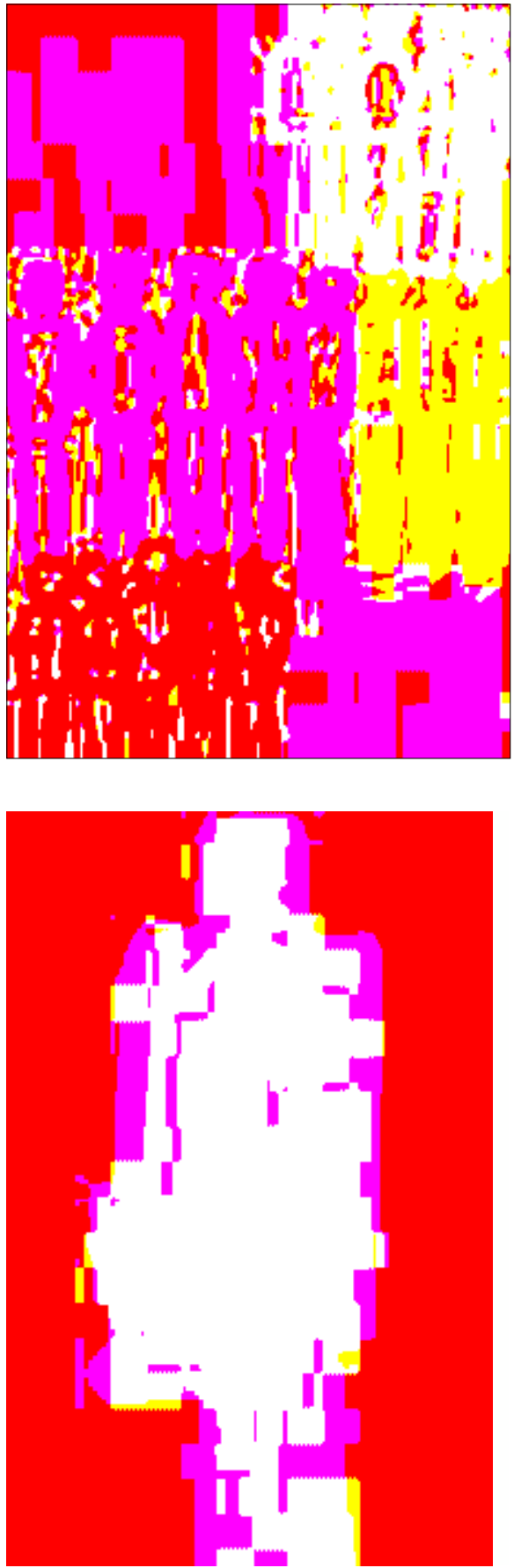


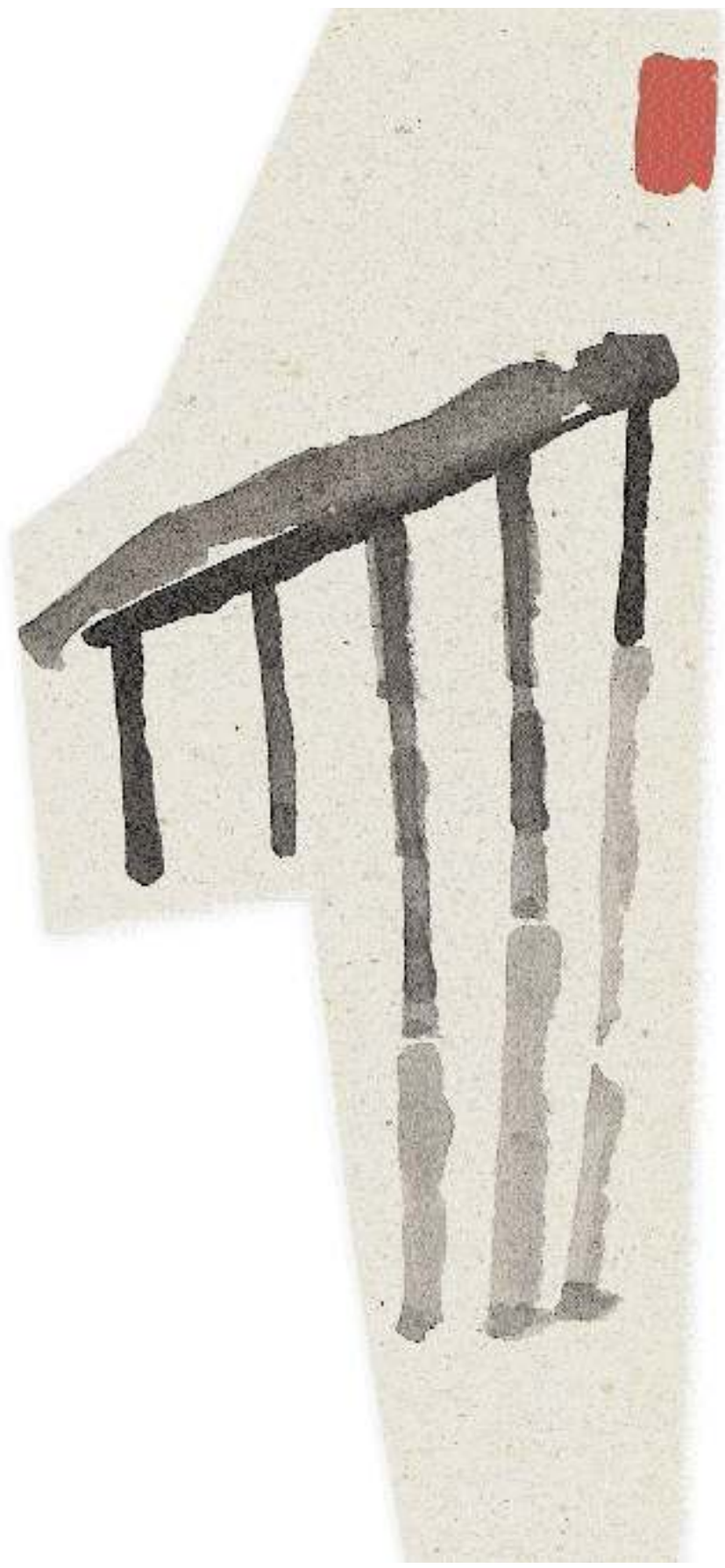

Mimmo Paladino, détail, illustration de l'lliade, Chant XXIV, 2001. 
3 Il semblerait que le phénomène soit lié à la naissance et à la diffusion des récits qui trouveront, dans les épopées homériques, leur forme la plus achevée. Ils permettent aux grandes familles d'Athènes de se rattacher aux modèles héroïques connus de tous.

4 Voir John Boardman, Aux origines de la peinture sur vase en Grèce (trad. CM.Diebold), Paris, Thames \& Hudson, 1999, (éd. originale en anglais, Londres, même éditeur, 1998), p. 24-28.

\section{Page 87}

Mimmo Paladino, illustration de l'Odyssée, Chant IX, 2001.
Je voudrais à présent analyser un dessin illustrant les funérailles d'Hector de la page 339 de l'lliade. Chez Paladino, la représentation de funérailles se manifeste dans une forme épurée, vidée du monde. Le défunt est seul sur son lit de mort, sous un soleil rouge carré. Il est davantage marqué par la solitude, le vide et l'absence des proches. En effet, ni les membres de la famille, ni les guerriers ne sont peints pour rendre un dernier hommage au défunt avant l'enterrement ou l'incinération. De même, rien ne nous indique l'activité guerrière du défunt.

En revanche, au temps d'Homère, le rituel attique se déroulait en plusieurs étapes, liées à l'idée de purification et de vénération du mort, permettant ainsi son passage d'un monde à l'autre. Par comparaison, je voudrais renvoyer à l'une des plus célèbres représentations de funérailles de la période de la Grèce archaïque. Le « Cratère géométrique attique », qu'on peut dater vers 760-750 av. J.-C., est conservé au Louvre et attribué au Maître du Dipylon qui semble avoir fixé le canon géométrique des figures humaines et animales, dessinées jusqu'ici de manière plus sommaire. Cette céramique représente un décor figuré très abondant. Au milieu d'ornements où domine le méandre, une scène de prothésis (exposition du mort sur son lit funèbre) rassemble la famille autour de sa dépouille. Le défunt est allongé sur le côté gauche. Les pleureuses avec leurs bras levés se lamentent. Enfin, chars, procession et bateaux de guerre évoquent l'activité guerrière et la noblesse héroïque du mort 4 .

Le traitement des scènes est abstrait. La figure humaine ou animale y apparaît sous une forme soumise au canon géométrique : le corps humain se dresse à la verticale. Le torse vu de face, par exemple, est un triangle, témoignant ainsi d'une réinterprétation de la silhouette qui prend volontairement ses distances avec la réalité.

Pour revenir à Mimmo Paladino, je dirais que la représentation humaine dans les illustrations d'Homère oscille entre figuration et abstraction.

Le deuxième exemple d'ombre que j'aimerais analyser est la représentation du cyclope. Paladino l'a illustré trois fois. À la page 97 de l'Odyssée, chant IX («Ah ! Le monstre étonnant ! II n'avait rien d'un bon mangeur de pain, d'un homme : on aurait dit plutôt quelque pic forestier qu'on voit se détacher sur le sommet des monts »), le Cyclope dévorant les victimes est représenté comme une masse informe (on ne peut distinguer les limites corporelles, entre mains et bras), masse boueuse (entre liquide et solide, soigneusement rendu par les qualités du lavis). Il ressemble davantage à un mont imprenable, occupant presque la totalité du dessin. La tête est cou- 


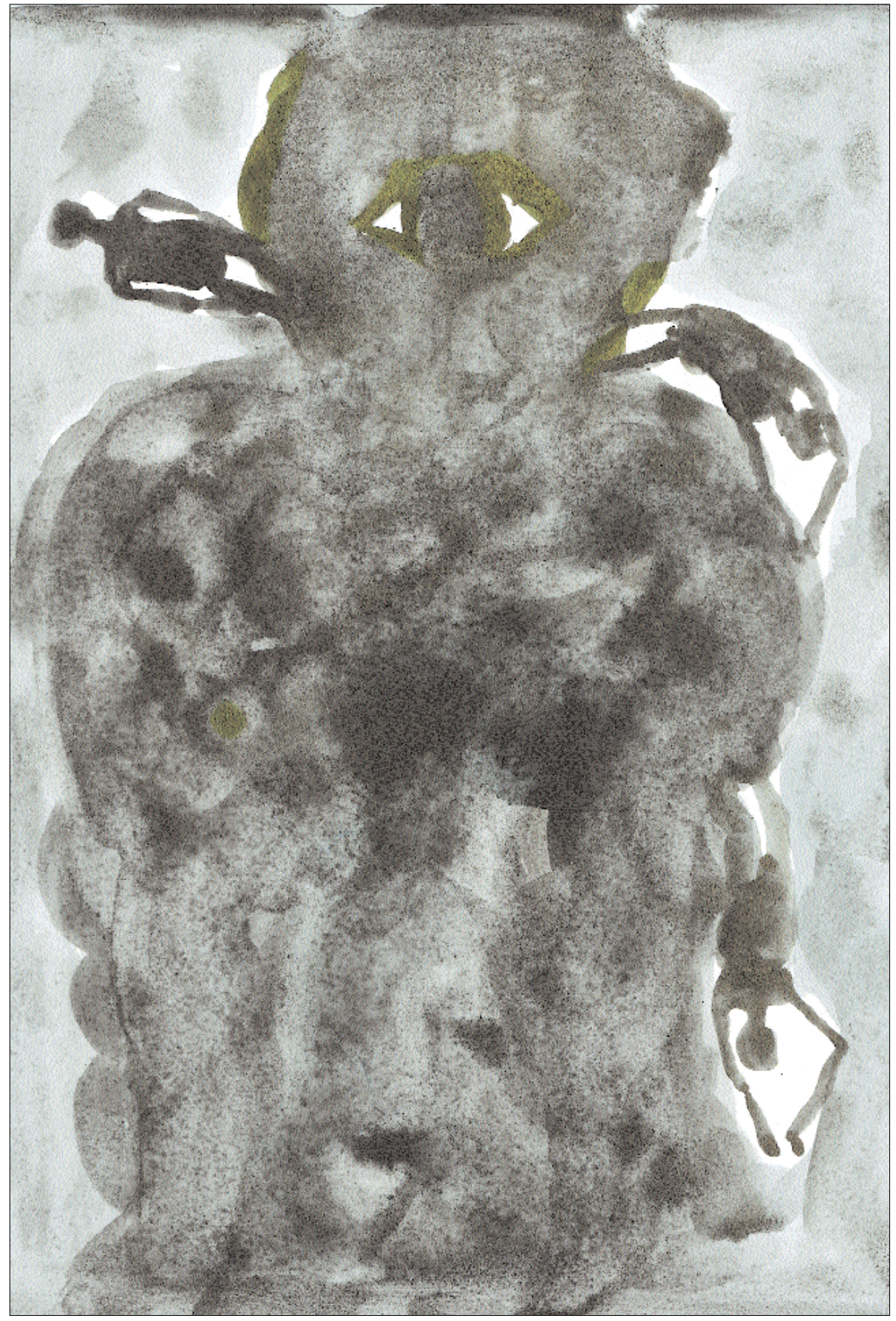




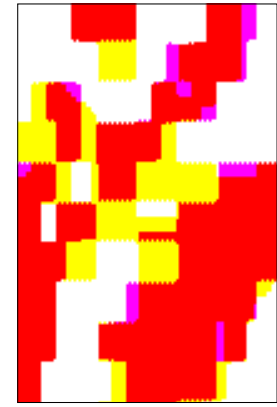

Détail, illustration p. 95.

pée, l'artiste ayant choisi un cadrage serré. Enfin, il est gigantesque par rapport aux ombres opaques des victimes humaines.

Le deuxième dessin à la page 103 correspond au passage du chant IX : « C'est ainsi qu'en son œil nous tenions et tournions notre pointe de feu, et le sang bouillonnant autour du pieu brûlant ". À nouveau, la délimitation, les contours du corps du Cyclope sont difficilement discernables. Seul l'œil unique, peint de face témoigne de sa présence. Ulysse, en guerrier veillant (qui nous rappelle le fameux dessin de Picasso représentant Don Quichotte), brandit sa lance touchant de sa pointe cet œil unique à abattre. On pourrait, indépendamment de l'épopée d'Homère, penser à la métaphore de l'artiste moderne qui tente de blesser, de rendre aveugle cet œil unique représenté par le point de fuite de la perspective. En effet, cette vision monoculaire a dominé l'histoire de la peinture depuis la Renaissance, en particulier depuis Alberti.

Enfin, la dernière représentation de Polyphème (page 107) montre ce dernier implorant l'aide de Poséidon. Il tend les deux mains vers le ciel. Son œil unique est à peine visible, réduit à la forme d'un trou blanc. En revanche, les mains sont grandes et disproportionnées, les doigts anormalement longs soulignant la lamentation et l'appel à la vengeance du dieu des mers. Ici le Cyclope apparaît seul, reprenant une apparence humaine, à la différence des deux précédentes illustrations. II n'incarne plus cette force monstrueuse, mais un fils qui implore l'amour et l'aide de son père.

Ces quelques exemples montrent l'importance accordée par Paladino à la question de l'ombre dans le champ artistique et philosophique. La Grèce ancienne a fondé notre perception de l'ombre et développé un savoir du royaume des ombres, à l'exemple des récits de la descente des Enfers comme rituels initiatiques pour Hercule, Persée...

\section{L'ombre à l'origine de la représentation}

L'ombre est à l'« origine » de deux mythes fondateurs de la peinture et de la théorie occidentale de la connaissance. Dans sa fameuse Histoire Naturelle (XXXV, 15), Pline situe « la question des ori-

Page 89 en haut Mimmo Paladino, illustration de l'Odyssée, chant IX, 2001.

Page 89 en bas Mimmo Paladino, illustration de l'Odyssée, Chant IX. gines de la peinture » non pas en Égypte, mais en Grèce. « Parmi les Grecs les uns disent qu'elle fut découverte à Sicyone, les autres à Corinthe, mais tous affirment qu'on commença par cerner d'un trait le contour de l'ombre humaine $»$.

Un peu plus loin dans son livre (XXXV, 43), Pline rapporte l'histoire de la fille d'un potier qui aurait fixé la silhouette de son bien-aimé, projetée sur le mur. Ainsi, pour conjurer l'absence prochaine de son 

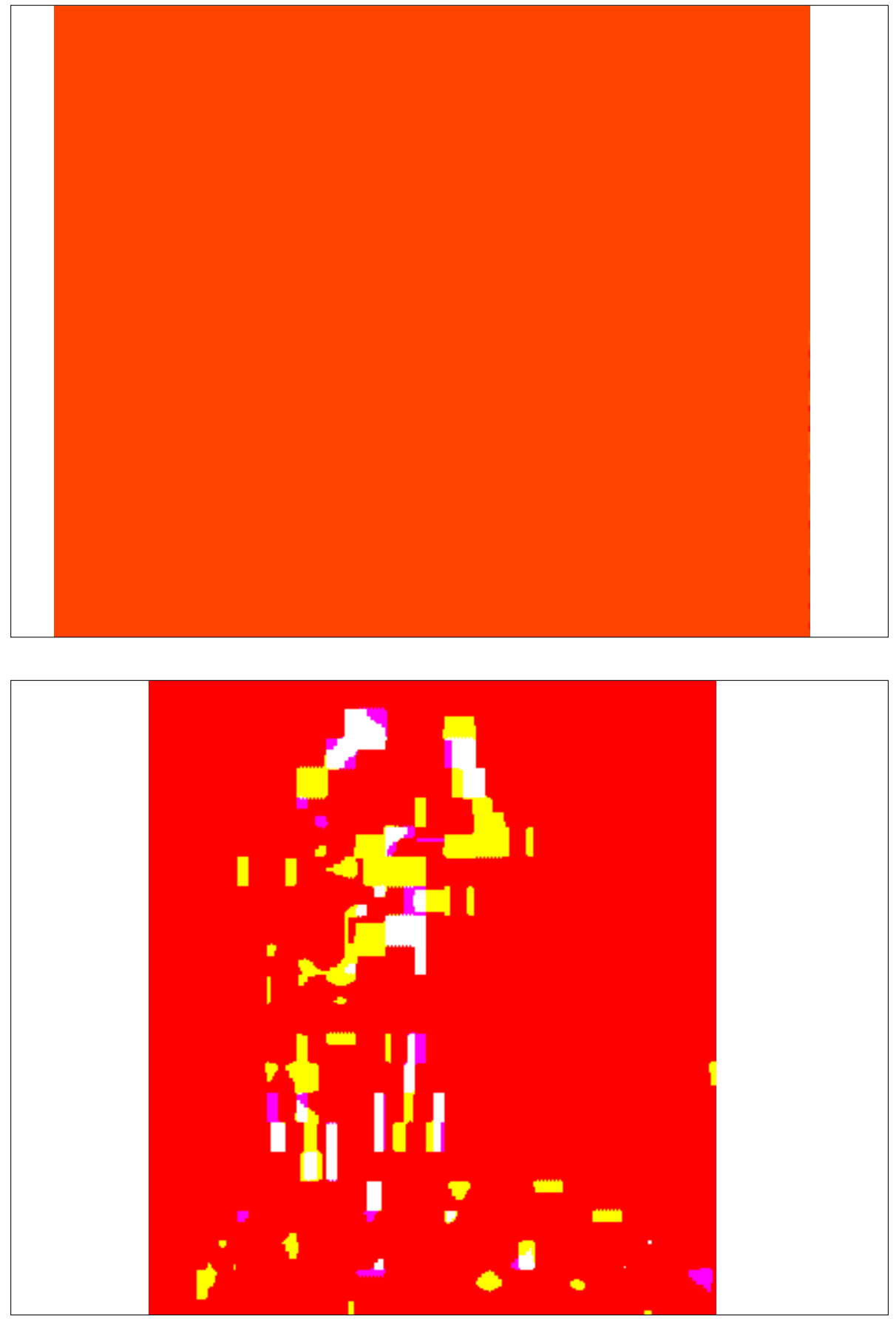
5 Platon, La République, trad. R. Baccou, GF Flammarion, Paris, 1966, VII 512 a-517b.

6 Victor I. Stoichita, Brève histoire de l'ombre, Droz, Genève, 2000, p. 31. amant, elle voulait en garder trace en fixant son ombre. C'est l'histoire marquée par le départ, où la douleur de la séparation est sublimée par un trait. La trace de l'ombre portée sur une surface d'inscription est un moyen pour conjurer l'absence, la mort. L'ombre opère une réduction du volume sur la surface. Ainsi les profils, les silhouettes sont le résultat d'une opération de projection. La première fonction de l'ombre serait d'être la matrice de l'image qui permet de rendre l'absent présent. La trace de l'ombre permet alors d'éterniser une présence sous forme d'image compensatoire. Ainsi l'être se réduit à une apparence, à un spectre. La silhouette tracée par la jeune femme n'est donc qu'un eidolon, une image sans substance qui est le double impalpable, immatériel de l'amant absent.

Dans ce sens, la légende de la fille du potier révèle qu'au regard du désir, la représentation se justifie comme " trace » avant la « ressemblance ». Ce récit de l'invention en " négatif » de la représentation peut être rapproché du mythe platonicien de la caverne. En effet, la pierre angulaire des deux mythes est l'ombre, une projection bidimensionnelle, une tache négative.

Avec L'Allégorie de la caverne, Platon serait le premier à envisager la question de l'image : " J'appelle image d'abord les ombres, ensuite les reflets que l'on voit dans les eaux ou à la surface des corps opaques, polis ou brillants, et toutes représentations semblables 5. " Rien, chez les Grecs anciens, n'est invisible. L'image n'est donc qu'une projection sur un écran qui joue de sa dimension illusoire. Elle est un double, une copie dégradée, une " image d'image ". Monoface, puisque aucun champ d'invisibilité ne se cache derrière l'écran-mur de la caverne, l'image n'est qu'un pâle dédoublement projeté sur une surface.

L'ombre, dans l'art contemporain, est omniprésente. Son autonomie (comme sujet à part entière et non plus comme forme définie à partir de l'objet) devient un signe de la modernité. En effet, l'ombre dialectise les notions du double et de l'altérité, l'autonomie et la menace de l'autre. Stoichita, dans sa Brève histoire de l'ombre, rapporte que « le stade du miroir, Lacan l'a dit, concerne principalement l'identification du je, tandis que l'ombre, elle, concerne principalement l'identification de l'autre. " L'historien de l'art en tire la conclusion que Narcisse est tombé amoureux de son image spéculaire et non pas de son ombre. « Et l'on comprend, également, chez Pline, la projection amoureuse de la jeune fille a pour objet l'ombre de l'autre (de l'amant) ${ }^{6}$. "

Ainsi, l'ombre, parce qu'elle est la doublure du réel, son image négative, renvoie au monde du simulacre et du double. Du latin 
simulacrum, le simulacre signifie une œuvre identique à son original ou une personne qui semble être le double de l'autre. Ce mot possède le même sens qu'eidòlon qui veut dire « ombre du mort ». Dans son article «Psuché : simulacre du corps ou image du divin », Jean-Pierre Vernant analyse, à partir de l'épopée homérique, les modes de représentation de l'invisible. Dans sa typologie des simulacres, il en distingue trois : le fantôme (phasma), le songe (oneiros) et le fantôme d'un mort (psuchê) ${ }^{7}$. Une fois décédés, les hommes deviennent des psuchai, c'est-à-dire des répliques. Ils mènent leur existence dans les ténèbres. Pour Platon, selon JeanPierre Vernant, ces êtres flottants sont des doubles, non plus corporels, mais spirituels, dotés de facultés psychiques, reliant ainsi les hommes aux dieux.

«Le fantôme, nommé le phasma, est créé par un dieu à la semblance d'une personne vivante, comme celui qu'Apollon fabrique "pareil à Énée lui-même et tel quant à ses armes" (lliade, v, 449453). Énée se trouve à l'abri dans Pergame où Apollon l'a déposé pour le soustraire à la bataille. C'est autour d'un simulacre que s'affrontent sans merci guerriers grecs et troyens également convaincus qu'il s'agit du héros en personne ${ }^{8}$. »

Parce qu'elle est fugace, l'ombre n'a d'existence que dans l'instant et s'apparente aux formes spectrales et aux mirages. Sans consistance, elle appartient à l'illusion et au fantasme. Sa fixation, sa trace répétée par le dessin est un " gain » d'iconisation par arrêt dans le temps.

À l'instar de l'ombre qui manifeste la perte et la persistance, l'image chez Paladino n'est jamais donnée dans son évidence. Dialoguant entre elles, les images mettent en relation avec des œuvres du passé et avec des notions théoriques. Ce montage retrame la temporalité des œuvres, provoquant ainsi l'apparition des formes survivantes. Ces images anciennes, oubliées, peuvent resurgir dans des processus visuels. Elles intensifient les images contemporaines, comme celles de Paladino. Ce dernier prend soin de montrer qu'il y a à deviner au-delà des apparences.

\section{La mémoire culturelle}

À partir du moment où l'histoire (histoire homérique entre autres) devient représentation, c'est le temps qui devient le sujet principal qui engloberait tous les autres. Et le temps, entendu comme ressassement de la picturalité, et éternel retour de la quête picturale, est soumis à de multiples distorsions. La relation, parfois conflictuelle, entre l'histoire et le temps est au cœur de la démarche de
${ }^{7}$ Jean-Pierre Vernant, «Psuché : simulacre du corps ou image du divin? ", dans Nouvelle Revue de Psychanalyse, n' 44,1991, p. 224.

8 ibid., p. 223. 
9 Gaston Bachelard, La poétique de l'espace, Paris, P.U.F., 1989, p. 6.

10 Il faudrait également réfléchir sur la place de la Trans-avant-garde italienne dans le contexte de l'art international des années quatre-vingt.
Paladino. Dans ses œuvres, il ne reste plus qu'une trame, une « trace mnésique " pour reprendre un terme de Freud.

Bien qu'on ait voulu voir l'œuvre de Paladino du côté de la citation, ne serait-il pas plus pertinent de la situer dans le registre de la réflexion ? Le recours à des formes chargées de sens et d'histoire n'a rien de nostalgique. Son art n'est pas citationnel, et n'est pas non plus une méditation solitaire sur l'histoire de la peinture. Privilégiant un art non mimétique, Paladino donne à voir des images spectrales, en particulier lorsqu'il peint des silhouettes et des ombres. Ses images archétypes sont comme des « résonances et du retentissement ": "Les résonances se dispersent sur les différents plans de notre vie dans le monde, le retentissement nous appelle à un approfondissement de notre existence ", écrit Bachelard dans La poétique de l'espace ${ }^{9}$. De quelles réminiscences les images de Paladino sont-elles porteuses?

L'image devient un réceptacle de figures, de motifs en mouvement, en déplacement. Ses "images survivantes » - Nachleben (l'« après-vivre ») pour reprendre le concept et le modèle culturel de l'histoire de Warburg qui a été analysé par Didi-Huberman, dans son dernier ouvrage L'image survivante - ont ce pouvoir de faire voir et de donner à revoir. L'après s'affranchit presque de l'avant, tout comme la forme s'affranchit presque de son contenu. Il s'agit donc de relativiser des notions comme celle de "tradition " car la transmission est complexe, anachronique et hybride. Elle est faite de processus inconscients, d'oubli, de sublimation et d'altération.

Dans cette perspective, les œuvres de Paladino apparaissent comme des réminiscences qui obligent le spectateur à ne plus être seulement devant une image unique ou devant un temps absolu, face à une image dense, dans un temps de la mémoire culturelle. Penser l'image comme un moment dynamique autorise à considérer le présent comme tissé de passés multiples. C'est ce qui expliquerait peut-être la " permanence » de certaines formes artistiques dans la longue durée de l'histoire de l'art.

En effet, il m'a semblé que les motifs des casques, masques et en particulier les silhouettes d'ombre (pour ne citer que ces quelques exemples car on pourrait de la même manière étudier d'autres motifs comme les chevaux, les attelages ou les bateaux) apparaissent comme des symptômes, c'est-à-dire des choses déplacées et anachroniques dans un temps de " crise ${ }^{\mathbf{1 0}}$ ". L'image qui est donnée à voir chez Paladino n'est donc pas la représentation d'un portrait, d'une composition, mais marque davantage une évocation, une présence. Ces images, vestiges d'un temps ancien, ressurgissent à travers les figures et les motifs récurrents tels que masques 
et casques. Certains éléments figuratifs ont disparu ou sont enfouis depuis longtemps, mais ils peuvent être repérés. Ce qui est rendu visible, ce sont des "survivances ", des réminiscences conceptuelles. L'œuvre de Paladino est une mémoire strate des formes. Le devenir de ces formes devant être perçu entre un désir d'identification, et une contrainte d'écart ou une altération : « La forme survivante, au sens de Warburg, ne survit pas triomphalement à la mort de ses concurrents. Bien au contraire, elle survit, symptomalement et fantomalement, à sa propre mort : ayant disparu en un point de l'histoire, étant réapparue bien plus tard, à un moment où, peutêtre, on ne l'attendait plus, ayant, par conséquent, survécu dans les limbes encore mal définis d'une "mémoire collective" ${ }^{11}$ ", écrit DidiHuberman.

Enfin, pour conclure, je dirais que se situer du côté de la survivance, c'est privilégier la transversalité des temps et non leur horizontalité, voire leur verticalité. Les œuvres de Paladino décrivent un autre temps, différé, anachronique même si ces traces sont ténues, à peine perceptibles. La survivance trouble et désoriente l'histoire. À travers ses deux cent deux œuvres, Paladino place le spectateur lui-même au cœur d'une épopée, faisant de sa trajectoire visuelle et intellectuelle une métaphore de la rencontre des différences.

Soko Phay-Vakalis
${ }^{11}$ Georges

Didi-Huberman,

L'image survivante.

Histoire de l'art et temps des fantômes selon Aby Warburg, Paris, Minuit, 2002, p. 67. 